\title{
Audio Information Camouflage Detection for Social Networks
}

\author{
Jiu Lou, Zhongliang Xu, Decheng Zuo*, Zhan Zhang and Lin Ye \\ School of Computer Science and Technology, Harbin Institute of Technology, Harbin, China
}

Sending camouflaged audio information for fraud in social networks has become a new means of social networks attack. The hidden acoustic events in the audio scene play an important role in the detection of camouflaged audio information. Therefore, the application of machine learning methods to represent hidden information in audio streams has become a hot issue in the field of network security detection. This study proposes a heuristic mask for empirical mode decomposition (HM-EMD) method for extracting hidden features from audio streams. The method consists of two parts: First, it constructs heuristic mask signals related to the signal's structure to solve the modal mixing problem in intrinsic mode function (IMF) and obtains a pure IMF related to the signal's structure. Second, a series of hidden features in environment-oriented audio streams is constructed on the basis of the IMF. A machine learning method and hidden information features are

OPEN ACCESS

Edited by: Shudong Li,

Guangzhou University, China

Reviewed by:

Chengyi Xia,

Tianjin University of Technology, China Longfei Wu,

Fayetteville State University,

United States

Chao Gao,

Southwest University, China

*Correspondence:

Decheng Zuo

zuodc@hit.edu.cn

Specialty section: This article was submitted to Social Physics,

a section of the journal

Frontiers in Physics

Received: 27 May 2021

Accepted: 20 July 2021

Published: 04 August 2021

Citation:

Lou J, Xu Z, Zuo D, Zhang Z and Ye L (2021) Audio Information Camouflage Detection for Social Networks.

Front. Phys. 9:715465

doi: 10.3389/fphy.2021.715465 subsequently used for audio stream scene classification. Experimental results show that the hidden information features of audio streams based on HM-EMD are better than the classical mel cepstrum coefficients (MFCC) under different classifiers. Moreover, the classification accuracy achieved with HM-EMD increases by 17.4 percentage points under the three-layer perceptron and by $1.3 \%$ under the depth model of TridentResNet. The hidden information features extracted by HM-EMD from audio streams revealed that the proposed method could effectively detect camouflaged audio information in social networks, which provides a new research idea for improving the security of social networks.

Keywords: social networks, machine learning, audio information camouflage, audio scene classification, emd

\section{INTRODUCTION}

Getting hot topics through social networks [1] and sharing news based on communities [2] have become the life style of modern people. Especially with the rise of we media technology in recent years, audio information has gradually become one of the main forms of information exchange in social networks. But it also brings a lot of security risks [3]. Using speech synthesis, interception audio stream for reediting and other methods to generate camouflage audio for fraud has become a new means of social networks attack. As a result of the rapid development of modern speech processing technology, people can easily edit "false" audio that cannot be easily distinguished by hearing, which makes it more difficult for people to distinguish the true and false audio in social networks. Therefore, the application of machine learning methods to mine hidden information in acoustic signals to identify authenticity and monitor risks $[4,5]$ has become a research hotspot in the field of acoustic signal processing. The special complex noise and sudden acoustic events in ambient background sounds are some of the important factors to judge audio authenticity. Furthermore, the frequency aliasing caused by complex noise and abrupt frequency changes caused by sudden acoustic 
events increases the difficulty of classifying environmental audio streams. Therefore, this paper proposes an audio environment hidden information feature extraction method based on HMEMD and aims to use the key factors contained in the special environment information to detect the authenticity of audio and solve the problem of audio fraud in social networks.

In the current deep learning framework, audio environment hidden information feature extraction methods are mainly divided into two categories: traditional feature representation [6-8] and automatic learning audio feature representation based on deep network $[9,10]$. MFCC [11], spectrograms, acoustic event histograms [12], and gradient histograms based on time-frequency learning [13] are the most commonly used traditional methods for acoustic feature representation. Acoustic event classification and detection is generally based on the spectral features of the MFCC [14]. The first team proposed a deep network for the TridentResNet series and used the snapshot method to filter the network classification results [15]. In addition to the aforementioned classical feature representation methods, deep neural networks (DNNs) can automatically learn audio features. The typical network models include the time-delay neural network [16], VGGISH-based embedding model [17], and the mixed feature extraction model based on DNN and convolutional neural network proposed by [18]. However, this end-to-end feature was not used by the first 30 teams in the DCASE tournament. This is because the end-to-end feature learning approach using deep networks directly requires a large number of evenly distributed data sets; however, in the real scene, the environmental sound source is complex, and the occurrence time and frequency of hidden acoustic events in environmental audio streams are not fixed and are often random and unpredictable. These scenarios enhance the mutagenicity and nonstationary properties of environmental acoustic signals and indirectly lead to the uneven distribution of various hidden acoustic events in datasets [19]. The result of the MFCC processing of acoustic signals is the mel-frequency. The mel-filter is designed according to the sensitivity of the human ear to frequency. Therefore, the MFCC achieves good results in speech and speaker recognition. However, in a nonspeech environment, ambient sound signals are nonstationary and hidden, and the sequence features of the MFCC lose a large amount of high-frequency hidden information and hidden information outside the hearing threshold range. Therefore, to improve the accuracy of hidden information mining in environmental audio streams, it is necessary to establish a more accurate time-frequency feature representation system that can further locate and analyze hidden acoustic events and ultimately improve the classification accuracy of environmental audio streams.

Environmental audio streams are typical nonlinear and nonstationary time-varying signals. Thus, they require timevarying filtering and decomposition technologies. Proposed by Norden E. Huang in 1998, empirical mode decomposition (EMD) is a signal processing method suitable for nonlinear unsteady time-varying signals [20].

However, the traditional EMD method has a few disadvantages, including mode aliasing and the inconsistency of IMF dimensions after signal decomposition. These drawbacks limit the application of EMD to acoustic signal processing. Modal aliasing causes the frequency distribution of some IMFs after signal decomposition to overlap. Hence, accurately estimating the IMF range of a certain frequency distribution is difficult. Dimension inconsistency may cause variations in the number of IMFs obtained from the decomposition of source signals with the same frame length; these variations will lead to the mismatch of the required eigenvector dimensions and hinder the subsequent signal analysis and processing [21]. In 2005, R. Deering and J. E. Kaiser proposed the ensemble empirical mode decomposition (EEMD) decision method [22], which attempts to solve the problem of mode aliasing by introducing Gaussian white noise into the signal to be decomposed. In EEMD, the attributes of Gaussian white noise should be adjusted artificially. However, the Gaussian white noise leaves traces in the IMF decomposed from the signal, thereby resulting in low signal restoration accuracy and extensive calculations. Timevarying filtering-based empirical mode decomposition (TVFEMD) uses the b-spline time-varying filter for mode selection and thus solves the problem of mode aliasing to a certain extent. However, TVF-EMD must calculate the cutoff frequency first, thus leaving the problem of dimension inconsistency unsolved [23].

By taking advantage of the time-frequency analysis of EMD, the problems of modal aliasing and frequency inconsistency in EMD must be resolved. Therefore, the current study consists of two parts. First, based on the traditional EMD, an improved heuristic empirical mode decomposition (HM-EMD) method is proposed. This method improves the purity of IMFs by adopting adaptive mask signals. With this method, the frequency domain distribution and IMF dimension can be stabilized and the inconsistency of the IMF dimension can be improved. The mask signals introduced in EMD can be obtained through heuristic learning and provide technical support for the feature extraction of hidden acoustic signals. On the basis of the mask signals, the hidden audio component features (HACFs) for audio stream recognition are constructed. According to the classification dataset Task-A [19] of the environmental audio stream in DCASE, hidden acoustic events, such as 'birdsong' and 'footsteps' in environmental audio streams, can be located and analyzed. The analysis results can be applied for multiple levels and multiple time scales of environment safety certification in audio streams. They can also be applied to other complex acoustic analyses and processing.

This work is divided into five parts. The second part mainly introduces the principle of HM-EMD. The signal processing flow and existing problems of the classical EMD algorithm are analyzed, and the principle of the HM-EMD algorithm is presented in detail. The third part describes the mining of hidden information in audio streams on the basis of the proposed HM-EMD. Specifically, environmental audio stream data are analysed, and the HACFs for hidden information in audio streams are designed according to the analysis results. The fourth part presents the classification of audio streams on the basis of HM-EMD. The experimental dataset is obtained from the low-complexity acoustic scene 
classification task provided by DCASE in 2020. The experimental results show that the proposed method can accurately extract and locate hidden acoustic events, thus improving the accuracy of audio stream classification. The fifth part summarises the characteristics of the proposed method and presents future research directions.

\section{HEURISTIC MASK FOR EMPIRICAL MODE DECOMPOSITION}

\section{Empirical Mode Decomposition Method Empirical Mode Decomposition}

EMD can decompose the original signal $\mathrm{x}(\mathrm{t})$ $(t \in N, N=\{0,1, \ldots \ldots n\})$ into a series of IMFs whose upper and lower envelopes have a mean value of 0 . This decomposition method does not need to preset basis functions (such as Fourier transform or wavelet analysis), but the IMFs should satisfy the following formulas:

$$
\begin{gathered}
\left|N u m_{\text {extream }}-N u m_{\text {cross }}\right| \leq 1 \\
\sum_{t \in N} B_{\max }(t)+\sum_{t \in N} B_{\min }(t)=0
\end{gathered}
$$

where Num $_{\text {extream }}$ is the number of extreme points of the data sequence and Num cross is the number of zero crossings; $B_{\max }(t), B_{\min }(t)$ are the upper and lower envelopes by cubic spline interpolation with the maximum and minimum points as the control points, respectively. Equation 1 represents the narrow-band constraint condition of the IMF, and Eq. 2 represents the local symmetry constraint condition. Algorithm description in Algorithm 1.

\section{Modal Aliasing of EMD}

The most significant disadvantage of EMD is mode aliasing. In mode aliasing, a single IMF contains signals of different frequencies or signals of the same frequency that appear in different IMF components. The typical mode aliasing phenomena are described as follows:

1) For multiple single-frequency signals, a mixed signal is an amplitude modulation (AM)-frequency modulation (FM) signal if the energy levels of the source signals are similar. When the frequency ratio is $\epsilon[0.5,2]$, the FM signal and AM signal overlap and the amplitude between the extreme values changes excessively. In such a case, the ordinary cubic spline function cannot easily and accurately fit any signal, resulting in the loss of local scale. This condition also leads to the mixing of multiple frequency domains in the IMF composition, such as signal $x_{1}(t)=\sin 2 \pi^{*} 2.4 \mathrm{t}+\sin 2 \pi^{*} 3.5 \mathrm{t}+\sin 2 \pi^{*} 7 \mathrm{t}$, the frequency ratio between two mixed signals is $1.45,2$ and 2.91 . There are two frequency ratio is $\epsilon[0.5,2]$. The EMD Results for $x_{1}(t)$ is shown in Figure 1. Figure 1A shows the IMFs of signal, which the corresponding FFT transform spectrum shows in Figure 1B. Mode aliasing can be seen in the FFT spectrum IMF1 and IMF2 in Figure 1B and abnormal mutation of the instantaneous frequency occurred in IMFs in Figure 1C.

2) Several different frequency signals are superimposed at different times, and the maximum value of the nonaliased part is missing, resulting in modal aliasing, such as EMD Results for signal $\quad x_{2}(t)=u(t, 0,2) * \sin 2 \pi * 2.4 t+\sin 2 \pi * 6 t+$ $u(t, 8,10) * \sin 2 \pi * 15 t$ shown in Figure 2 where

$$
u t, t_{1}, t_{2}=\left\{\begin{array}{lr}
1 & t \in\left\{t_{1}, t_{2}\right\} \\
0 & \text { others }
\end{array} .\right.
$$

The frequency ratios in $x_{2}(t)$ are all out of $[0.5,2]$, but there sin $\left(2 \pi^{\star} 2.4 \mathrm{t}\right)$ start at time $2 \mathrm{~s}$ and the $\sin \left(2 \pi^{\star} 15 \mathrm{t}\right)$ end at time $8 \mathrm{~s}$ which lead to the mode aliasing as shown in Figure $2 \mathbf{B}$. The abnormal mutation of the instantaneous frequency occurred in IMFs in Figure 2C.

3) Mode aliasing also occurs when the distribution of extreme points in a window is not uniform even if Eqs 1, 2 are satisfied, such as signal $x_{3}(t)=u t, 0,2 * \sin 2 \pi * 2.4 t+\sin 2 \pi * 3.5 t+u t, 8,10 * \sin 2 \pi * 7 t$. The model aliasing can be seen in FFT spectrum of IMFS in Figure 3B. The negative frequency in Figure $\mathbf{3 C}$ is caused by the large fluctuation of the IMF amplitude.

When the frequency interval between multiple signals is too small or there is noise, the local extremum will jump many times in a very short time interval. The local extremum as control points for cubic b-spline interpolation in the process of EMD. The cubic b-spline interpolation resulting in the spectrum envelope will be fluctuated if the extreme value loss or extreme value distribution is inconsistent. This condition can adversely affect the spectral envelope. At this time, the time-domain signal does not meet the narrow-band requirements of IMF decomposition, resulting in mode aliasing. Therefore, the absence of extremum is an important cause of mode aliasing in EMD calculation. The different causes of the lack of extreme values require different processing methods. The causes of missing extreme values can be divided into two categories. One is the uneven distribution of extreme values in the analysis window caused by signal concealment at a certain time (Figure 2). The key to dealing with this type of modal aliasing is to

\section{ALGORITHM 1 | EMD decomposition to obtain an IMF}



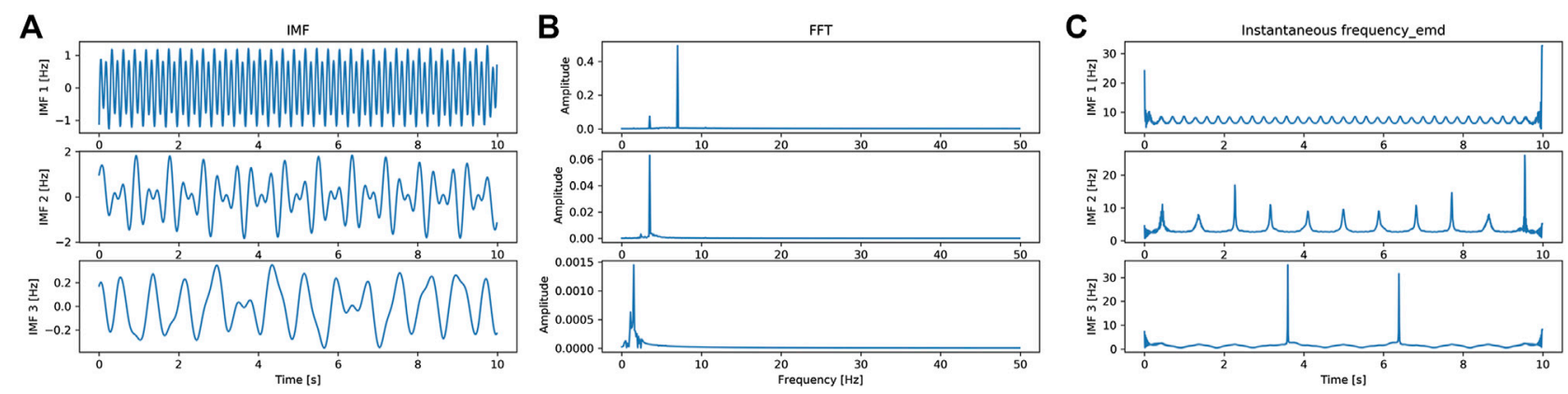

FIGURE 1 | EMD results for signal $x_{1}(t)$, (A) IMFs of $x_{1}(t)$; (B) FFT spectrum of each corresponding IMF; (C) Instantaneous frequency of each IMF.


FIGURE 2 | EMD results for signal $\mathrm{x}_{2}(\mathrm{t})$, (A) IMFs of $\mathrm{x}_{2}(\mathrm{t})$; (B) FFT spectrum of each corresponding IMF; (C) Instantaneous frequency of each IMF.
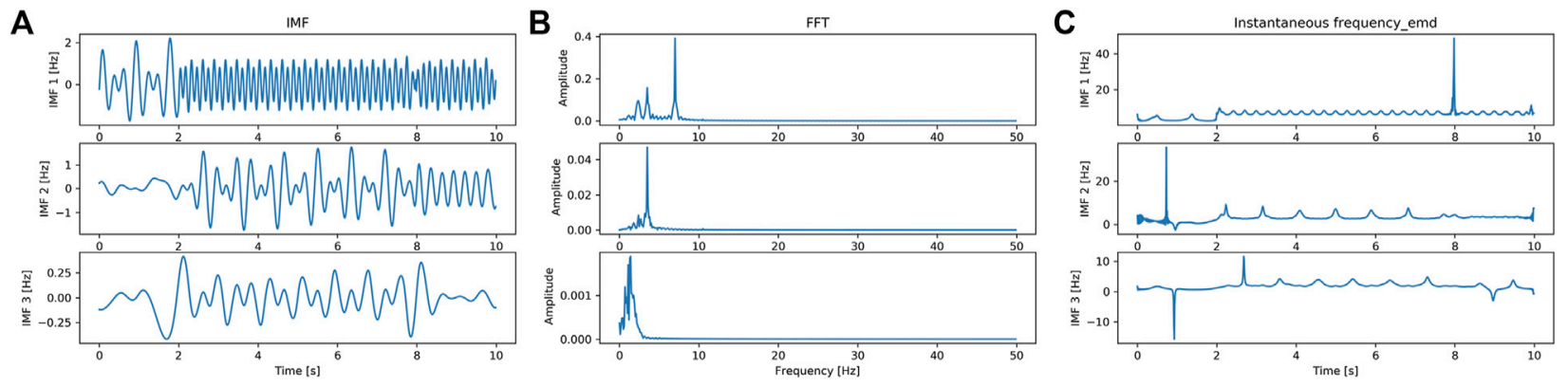

FIGURE 3 | EMD results for signal $x_{3}(t)$, (A) IMFs of $x_{3}(t)$; (B) FFT spectrum of each corresponding IMF; (C) Instantaneous frequency of each IMF.

determine the time when the signal concealment occurs. If the analysis is conducted according to the time point of concealment, then aliasing will not occur. The other category involves signal spectrum aliasing, which can be addressed by adding mask signals; that is, by creating a mask signal $\mathrm{s}(\mathrm{T})$, we can derive the following:

$$
\begin{aligned}
& x_{+}(t)=x t+s t \\
& x_{-}(t)=x t-s t
\end{aligned}
$$

For $x_{-}(t)$ and $x_{+}(t)$, EMD is performed to obtain the natural mode functions $r_{I M F-}(t)$ and $r_{I M F+}(t)$, respectively. The final IMF is defined as follows:

$$
r_{I M F}(t)=\frac{r_{I M F+}(t)+r_{I M F-}(t)}{2}
$$

However, signal mode aliasing in practical applications cannot be attributed to a single factor (Figure 3). It usually includes hiding and spectrum aliasing. Therefore, it can be considered to determine the time of signal concealment. Then, a mask signal is introduced to the period of concealment to perform mode decomposition. Therefore, the current work proposes the HM-EMD method. This method maximises the use of the intrinsic properties of signals to construct variable analysis windows and mask signals that can adapt to a variety of signal 
contents. The principle and implementation process are described herein.

\section{Heuristic Mask Signals Basic Principle Analysis}

The signal properties need to be established prior to EMD. A time-varying FM/AM model can be used to express any nonstationary signal; that is,

$$
x(t)=A t \sin (\omega(t))
$$

where a $(\mathrm{T})$ is the envelope function and $\omega(\mathrm{T})$ is the phase function. The analytical signal is

$$
z(t)=\mathrm{x} t+j H[x(t)]
$$

Here, $H[\cdot]$ denotes the Hilbert transform. We calculate the instantaneous phase $\omega(t)=\arctan \frac{H[x(t)]}{x(t)}$ and instantaneous frequency $f_{I F} t=\frac{1}{2 \pi} \frac{d[\omega(t)]}{d t}$. Using Hilbert transform, we can separate the AM and FM components of the IMF to achieve the purpose of modal separation.

For the single component mode, the instantaneous frequency $f_{I F} t$ should be nearly linear, while the variation range of $\omega(t)$ should be considerably small. When mode aliasing occurs, $f_{I F} t$ should clearly change without consideration of the end points. Especially, for hidden components, a jump of $f_{I F} t$ occurs at the time point of concealment, as shown in Figures 2D, 3D. We constructed a variable analysis window according to the time-frequency characteristics of instantaneous frequency. Then, we divided the signal into several parts.

If $f_{I F} t$ of the segmented signal is still unstable, then the modal separation problem can be transformed into the $\frac{d[\omega(t)]}{d t}$ minimisation problem, in which the bandwidth of $\sin (\omega(t))$ is minimised. The bandwidth calculation method for nonstationary signals can be obtained by the Carson rule:

$$
B W_{A M-F M}=2\left(\Delta f+f_{F M}+f_{A M}\right)
$$

where $\Delta f$ is the deviation of the instantaneous frequency from its mean value and $f_{A M}$ and $f_{F M}$ denote the frequencies of the AM and FM signals, respectively. We can make $\Delta f=0$ to minimise the bandwidth. In other words, the decomposition frequency of each IMF is expected to be equal to the centre frequency of the instantaneous frequency, that is, equal to the mean value of the instantaneous frequency $\overline{f_{I F}} t$. Then, a mask signal with the same frequency as $\overline{f_{I F}} t$ can be selected and the number of IMFs required can be determined.

\section{Algorithm Description}

The HM-EMD algorithm comprises the following steps: variable analysis window construction and mask signal construction.

1) Variable Analysis Window Construction.

The jump point $t_{i}$ should be picked such that Eq. 9 is satisfied:

$$
\left(\left|f_{I F}\left(t_{i}\right)-f_{I F}\left(t_{i+1}\right)\right|+\left|f_{I F}\left(t_{i-1}\right)-f_{I F}\left(t_{i}\right)\right|\right)>\mu_{\Delta f_{I F}(t)}+\rho \varepsilon_{\Delta f_{I F}(t)}
$$

where $\Delta f_{I F}(t)$ is the difference in instantaneous frequencies at $t_{i}$, $\mu_{\Delta f_{I F}(t)}$ is the mean value of $\Delta f_{I F}(t)$ at all time points, $\varepsilon_{\Delta f_{I F}(t)}$ is the variance and $\rho$ is the variable parameter. The original signal is divided into two parts by the time division points $t_{i}$ and decomposed by EMD independently.
2) Mask Signal Construction.

The sine signal is a common form of a mask signal, and its amplitude and frequency should be determined. As analyzed in Empirical Mode Decomposition Method, the frequency is determined as the average instantaneous frequency $\overline{f_{I F}}$. Hence, the amplitude is also determined as the mean value $\overline{A_{I F}}$ of the instantaneous amplitude. Then, the mask signal st is defined as

$$
s t=\overline{A_{I F}} \sin 2 \pi \overline{f_{I F}} t
$$

$\overline{A_{I F}}=\frac{1}{n} \sum_{t=1}^{n} \sqrt{r_{I F}(t)^{2}+H\left(r_{I F}(t)^{2}\right)}$ and $\frac{1}{n} \sum_{t=1}^{n} \frac{d}{d k}\left(\arctan \frac{H\left(r_{I F}(t)\right)}{r_{I F}(t)}\right)$.

Then, the IMFs can be refreshed by Eqs. 3-5, in which the number of IMFs are determined by $\overline{f_{I F}}$ and $f_{c}$ is the sampling frequency. The algorithm flow is as follows Algorithm 2.

\section{HM-EMD-BASED ACOUSTIC SCENE CLASSIFICATION}

\section{Acoustic Scene Signal Analysis}

When processing the original signal with HM-EMD, the variable analysis window and mask signal are used to intervene the decomposition of the original signal. The frame length is selected according to the frequency structure of the signal itself, while the frequency domain components corresponding to each IMF are relatively independent, which provides higher interpretability of the features. The instantaneous frequency and amplitude of each IMF also contain all information of IMF components, which means that the instantaneous frequency and amplitude of all IMF components contain most of the information of the signal to be analyzed, and can be directly used as the basic characteristics of the signal. Figure 4 shows the time-domain waveforms of some typical IMFs with hiding acoustic events in the ambient audio stream, in which only the most significant one of all IMF waveforms is shown. It can be seen that the time-domain waveform characteristics of these events are very obvious, the extreme value and over average rate are very different, and they are distributed in low, medium and high frequency bands, such the airport luggage roller in Figure 4A and Metro rail joint collision in Figure 4B are low frequency, steps in Figure 4D and tram acceleration in Figure 4F are medium frequency, chirm in Figure 4C, vehicles from far to near in Figure $4 \mathrm{E}$ are high frequency. Therefore, this paper proposes a full band IMF hiding component features, which can distinguish them well, to effectively improve the effect of ambient audio stream recognition algorithm, the feature calculation method is shown in Mutagenic Component Features.

\section{Mutagenic Component Features}

Figure 4 shows various hidden components in the acoustic scene data. On the one hand, the hidden components cause a significant interference to the signal spectrum, thereby greatly affecting the ambient audio stream recognition effect based on traditional spectrum features (such as MFCC). On the other hand, the types and characteristics of hidden components corresponding to different ambient audio streams also exhibit significant differences. These hidden components are closely related to the types of acoustic events. The features constructed on the basis of hidden 
Algorithm 2 | Heuristic empirical mode decomposition with a masking signal

Heuristic Empirical Mode Decomposition with a Masking Signal

Input: Signal, Supposed IMF number, input: Signal $x(t)$, Supposed IMF number i

output: Intrinsic Mode Function, IMF

$1 x_{1}(t)=x(t), i=1 ;$

2 Get the first IMF of the signal residual $x_{i}(t)$, calculate the mean and variance of $\Delta f_{I F} t$, use Eq. 8 to determine whether there is a hiding jump point, variable analysis window is constructed according to the hiding jump point and $x_{i}(t)$ is segmented.

3 Construct mask signal for each $I M F_{i}: s_{i} t=\overline{A_{I F}} \sin 2 \pi \overline{f_{I F}}$ t;

4 Do EMD on $x_{i+} t=x_{i}(t)+s_{i} t$ and $x_{i-} t=x_{i}(t)-s_{i} t$, get the first IMF $r_{M F_{i+}}(t)$ and $r_{I M F_{i-}}(t)$;

5 Let $r_{I M F_{i}}(t)=\left(r_{I M F_{i+}}(t)+r_{I M F_{i-}}(t)\right) 2$, and splice all the divided pieces.

$6 \mathrm{i}=\mathrm{i}+1, x_{i}(t)=x_{i-1}(t)-r_{I M F_{i}}(t)$, return to step2, until $\overline{f_{I F}} t<\frac{f_{c}}{2 i}$, or no new IMF is required;

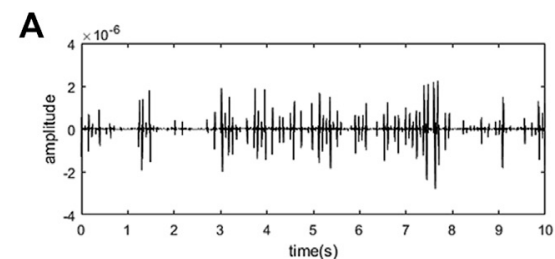

D

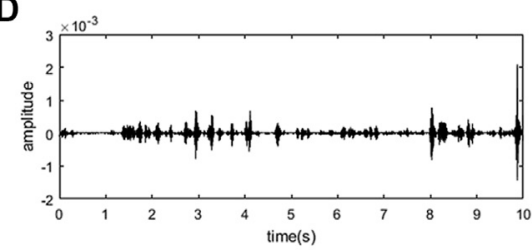

B



E

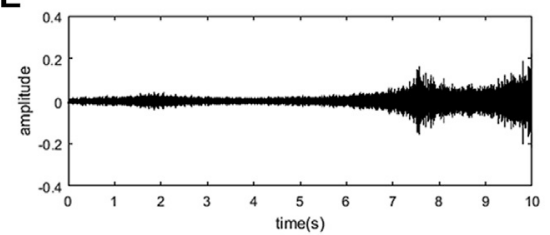

C

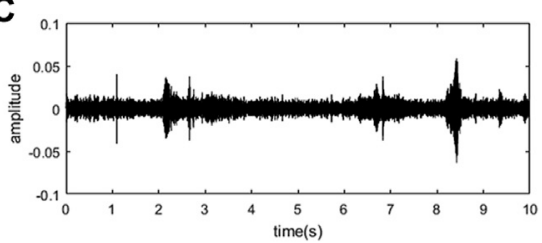

$\mathbf{F}$

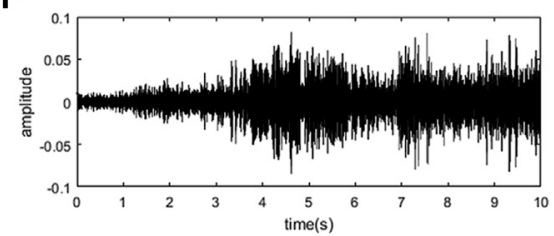

FIGURE 4 | IMF waveforms with significant hidden components in different environments of the audio stream, (A) Airport luggage roller-IMF16, low freq; (B) Metro rail joint collision-IMF14, low freq; (C) Chirm-IMF1, high freq; (D) steps-IMF10, medium freq; (E) Vehicles from far to near-IMF1, high freq; (F) Tram acceleration-IMF4, Medium and high freq.

components can help to distinguish ambient audio streams. For a hidden component, its frequency, amplitude and change mode information can effectively reflect its essential attributes. Almost all of such information can be reflected by the envelope shape of the IMF obtained by decomposition. Therefore, we design a set of HACFs. Based on the IMF decomposed by HM-EMD, the features extract the relevant information of hidden components, including the shock intensity feature $\mathrm{SH}$ and over-average feature average crossing rate (ACR).

1) Shock intensity feature $(\mathrm{SH})$ :

$$
S H_{\text {maxj }}=\max \left(r_{I M F j}^{u p}(t)\right) \text { and } S H_{\operatorname{minj}}=\min \left(r_{I M F j}^{u p}(t)\right)
$$

where $\max \left(r_{I M F j}^{u p}(\mathrm{t})\right)$ is the upper limit of the signal amplitude in the jth IMF and min $\left(r_{I M F j}^{u p}(\mathrm{t})\right)$ is the lower limit. Both limits represent the change intensities of the hidden components relative to the steady components for measuring the changes in signal amplitude. As the sum of the mean values of the upper and lower envelopes of the IMF is 0 , the signal is symmetrical along the time axis, and the information carried by the upper and lower envelopes are almost the same. Therefore, a one-sided envelope is enough to ensure the consistency of the symbols of the two values. The superscript means that the upper envelope is used for calculation.
2) ACR feature:

$$
\begin{aligned}
A C R_{i}= & \frac{1}{2 T} \sum_{i=2}^{T} \mid \operatorname{sgn}\left[r_{I M F j}^{u p}(t)-\overline{r_{I M F j}^{u p}(t)}\right]-\operatorname{sgn}\left[r_{I M F j}^{u p}(t-1)\right. \\
& \left.-\overline{r_{I M F j}^{u p}(t)}\right] \mid
\end{aligned}
$$

ACR features can express the number of times the upper envelope of an IMF passes through its mean point, that is, the number of times the IMF's upper envelope (time domain amplitude) fluctuates significantly. If the value is large, the IMF amplitude frequently fluctuates near the mean value. For ambient audio stream recognition application scenarios, if the value is greater than a certain threshold $(10 \mathrm{~Hz}$ or above), the data may not have obvious and meaningful hidden components and the change of the upper envelope near the mean value is only the normal fluctuation of the acoustic signal itself. If the value is less than the threshold, the data may contain significant hidden components, and one-half of the zero crossing frequency is the frequency of the hidden components.

\section{Ambient Audio Stream Classification}

The ambient audio stream classification process based on HM-EMD is shown in Figure 5. HM-EMD is used to obtain the IMF set of the 


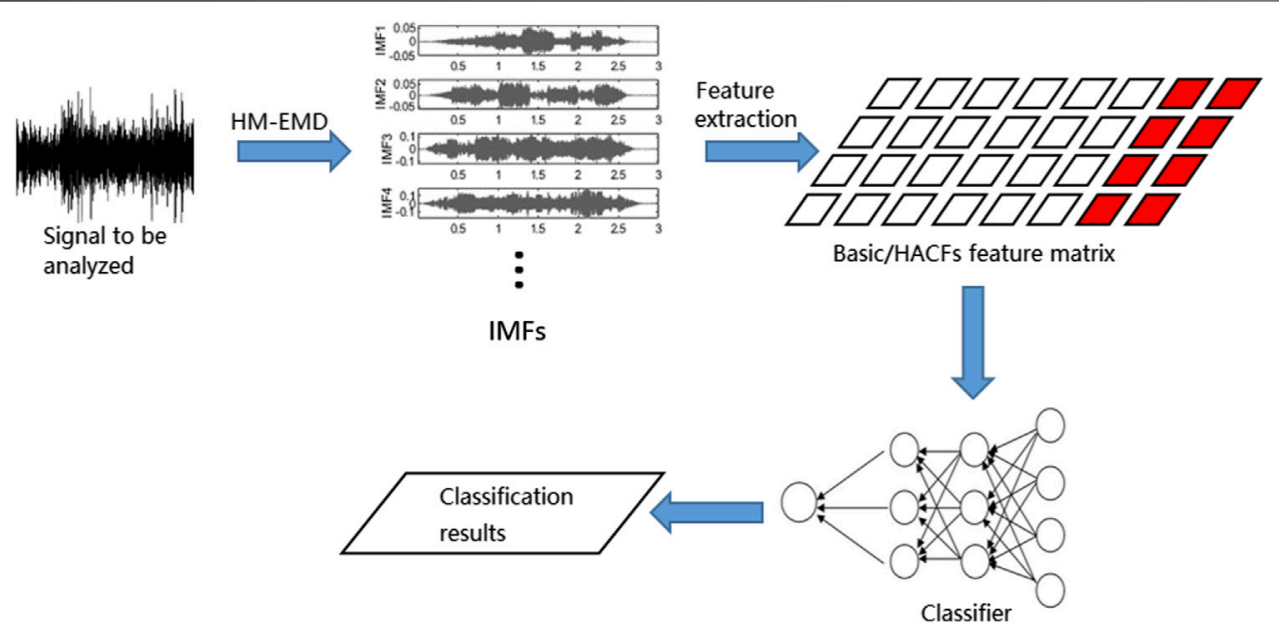

FIGURE $\mathbf{5}$ | Ambient audio stream classification flow chart based on heuristic mask empirical mode decomposition.

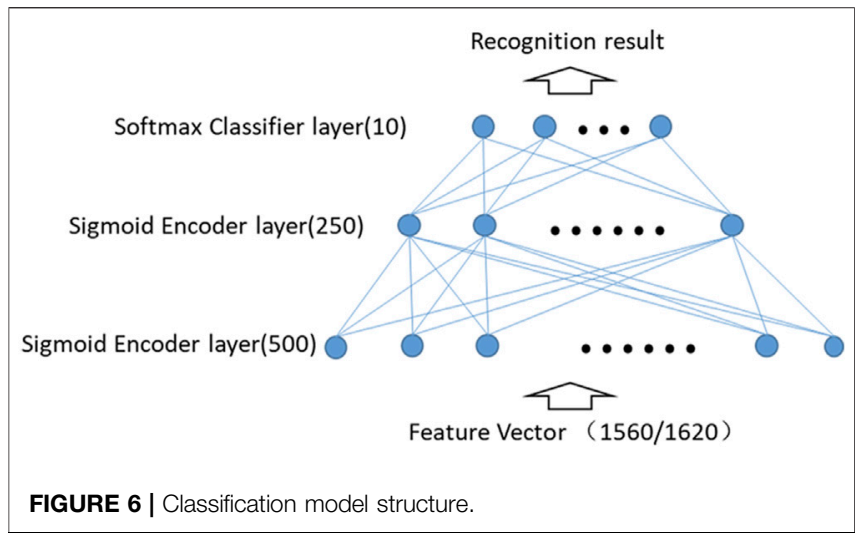

signal to be analyzed. Then, the following basic features are extracted: instantaneous frequency, instantaneous amplitude and the HACFs proposed in this work. Organised as a feature matrix, the features are input into the classifier to obtain the final recognition result. We select the neural network model for the classifier. The network structure is shown in Figure 6. To prove the effectiveness of the features, we select a three-layer neural network model. The first two layers use a sigmoid function as the activation function. First hidden layer has 500 and second hidden layer has 250 neurons. The output layer uses a softmax classifier and has 10 neurons. The experimental results show that the feature system still shows satisfactory results even with the use of a simple classification model. The specific experimental results and analysis are presented in the next section.

\section{EXPERIMENTS AND RESULTS}

\section{Experimental Setup}

We verify the results of this work from two aspects: the validity of modal separation and the validity of the HM-EMD features for environmental audio stream classification. The experiments use Python language, the deep learning framework uses the PyTorch framework, and the data set uses the task $1 \mathrm{~A}$ audio scene classification dataset in DCASE competition.

\section{Validation of Modal Separation}

A nonlinearity index is defined in Eq. 13, and it measures the stability of the decomposition results. The larger the DN is, the greater the nonlinear degree is, indicating the more unstable components; the verification data are the mixed signals of the three modes in Figures 1-3.

$$
D N=\left[\frac{1}{n} \sum_{t=1}^{n}\left(\frac{f_{I F} t-\overline{f_{I F}} t}{\overline{f_{I F}} t}\right)^{2}\right]^{1 / 2}
$$

\section{Validation of the Features of HM-EMD for the Classification of Ambient Audio Streams}

The data used in the experiment come from the TASK1A dataset of DCASE [19]. Task1A that is to classify the acoustic scene with multiple devices. The dataset contains data on ten cities and nine devices, that is, three real devices $(\mathrm{A}, \mathrm{B}, \mathrm{C})$ and six simulated devices (S1-S6). The dataset has good annotation, including three different types of indoor, outdoor and traffic. It also has ten different ambient audio streams, namely, airport, shopping mall, metro, metro station, pedestrian, street traffic, tram, park and public square and bus. The acoustic data span a total of $64 \mathrm{~h}$, with $40 \mathrm{~h}$ used in dataset training and with $24 \mathrm{~h}$ used in verification. Each audio segment is $10 \mathrm{~s}$ long, and the sampling rate is $44.1 \mathrm{kHz}$.

To verify the effectiveness of designing a series of features based on HM-EMD, we use a basic HM-EMD feature matrix and a basic features + HACF matrix as the input parameters of the classifier. Specifically, the number of mask EMD reference IMFs is 20, HMEMD basic feature is $2 \mathrm{D}$ and HACFs is $3 \mathrm{D}$, which number of dimension is $20 \times 3$. The audio frame length is $0.5 \mathrm{~s}$, and the interframe overlap is $0.25 \mathrm{~s}$, the total number of dimensions is $39 \times$ $20 \times 3=2340$. The classical MFCC are selected as the contrast features; they include 13 dimensional MFCCs and delta features. The 

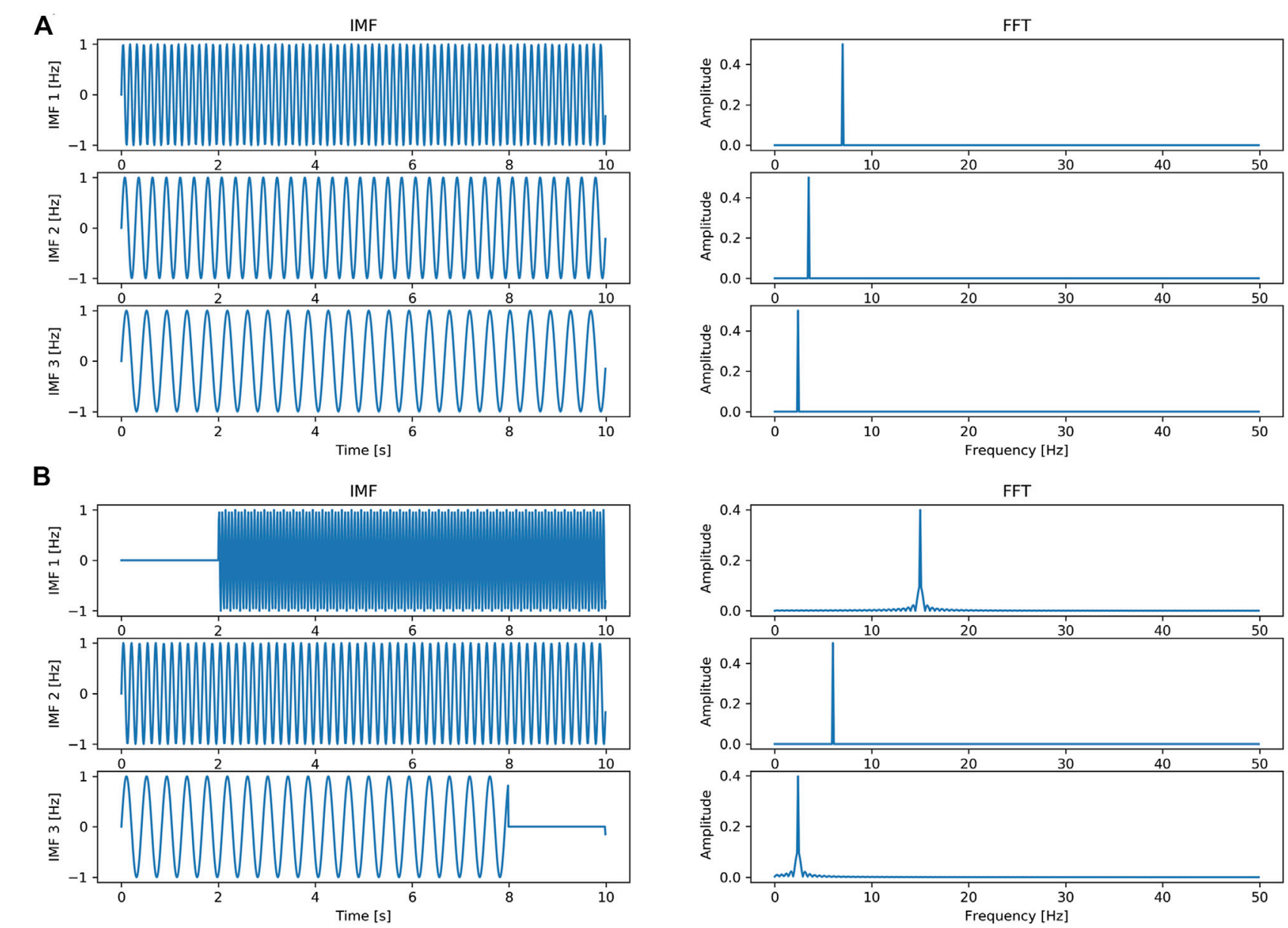

C
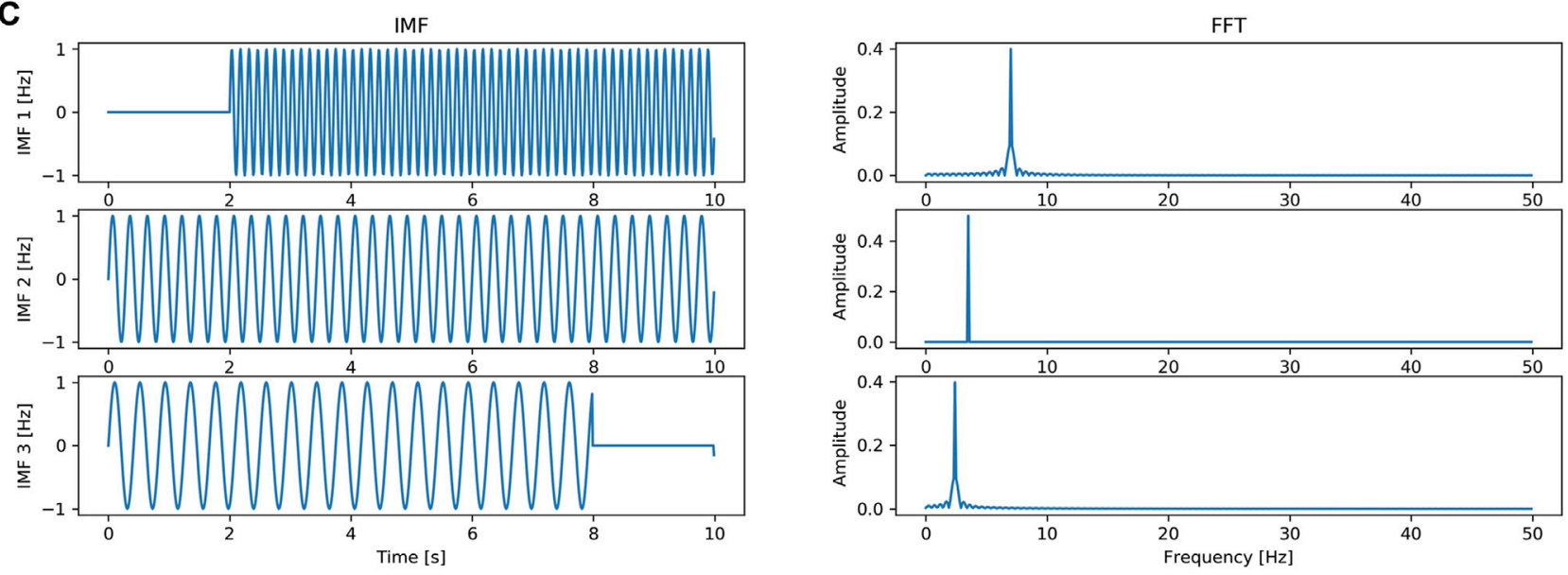

FIGURE 7 |HM-EMD results of $x_{1}(t), x_{2}(t), x_{3}(t),(\mathbf{A})$ IMFs of $x_{1}(t)$ and the FFT spectrum of each corresponding IMF; (B) IMFs of $x_{2}(t)$ and the FFT spectrum of each corresponding IMF; (C) IMFs of $x_{3}(t)$ and the FFT spectrum of each corresponding IMF.

total number of dimensions is 39 , and the audio frame length is $40 \mathrm{~ms}$. The specific experimental results are described herein.

After setting the characteristic parameters, we conducted the test according to the process designed in Figure 5. We trained the classifier parameters with the training dataset and tested them with the test set.
Results and Analysis

Effectiveness Analysis for Modal Separation

By comparing the traditional EMD results, we can see DNHM-EMD/DNEMD $<1$ for any given case. Hence, the IMF processed by the HM-EMD method has the lowest 

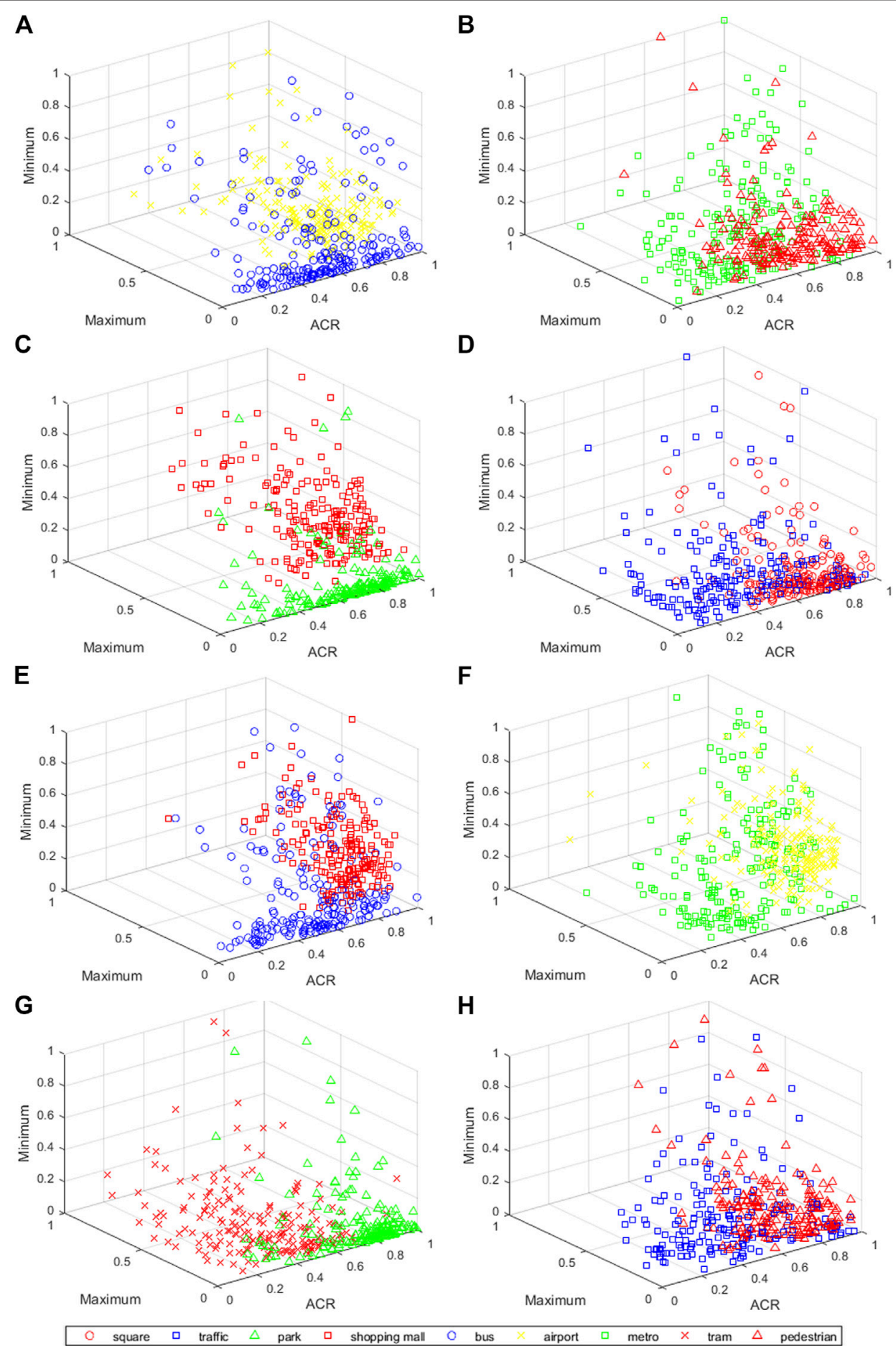

FIGURE 8 | HACFS feature distribution in 3D space, (A) IMF1 of bus \& airport; (B) IMF1 of metro \& pedestrian; (C) IMF1 of park \& shopping mall; (D) IMF1 of square \& traffic; (E) IMF2 of bus \& shopping mall; (F) IMF2 of met; (G) IMF2 of tram \& park; (H) IMF2 of traffic \& pedestrian.

nonlinearity; that is, the IMF has a high purity and is close to the blind separation result under an ideal state. The separation result is shown in Figure 7. From the FFT spectrum corresponding the IMFs of $x_{1}(t), x_{2}(t)$ and $x_{3}(t)$ in Figures 7A-C, we can see that the mode aliasing was solved and each IMF was pure. The features based on this high-purity 


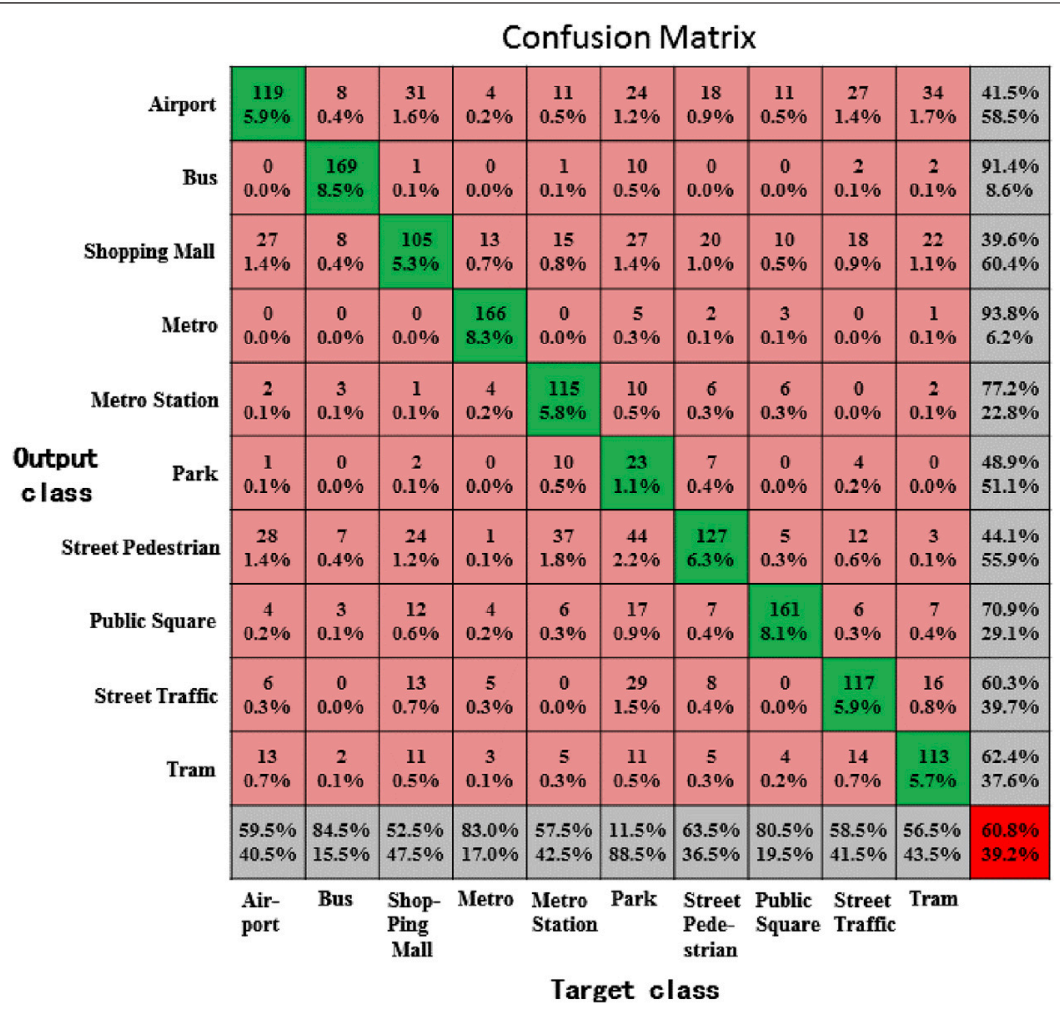

FIGURE 9 | Recognition results of basic instantaneous frequency and instantaneous amplitude features.



FIGURE 10 | Recognition results of basic features and HACFs features. 
IMF signal can effectively characterize the subtle changes in the signal components in the time and frequency domains. Hence, the method is suitable for all types of acoustic correlation analyses and recognition, especially for the recognition of ambient audio streams with hidden acoustic events.

\section{Based on HM-Feature Validity of EMD}

HACFs can be used to identify the hidden components in IMFs and are thus of great significance for ambient audio stream recognition. We verified the discrimination ability of HACFs in different scenarios in Figure 8. The figure shows the scatter projection of some hidden component features in the threedimensional space. Even the three-dimensional features in a single IMF have a strong scene discrimination ability. HACFs show good discrimination ability among different ambient audio stream categories and thus provide technical support for subsequent ambient audio stream classification.

We use the simple classifier shown in Figure 6 to classify and recognize the environmental audio streams in different scenes based on HM-EMD basic feature and basic + HACFs feature respectively, and then use the confusion matrix to represent the recognition accuracy in each scene. The vertical axis represents the classification output, and the horizontal axis represents the annotation result, as shown in Figures 9, 10. As can be seen from Figure 9, the average recognition rate for all scenes is $60.8 \%$, and the average recognition rate increases to $71.5 \%$ in Figure 10 after adding HACFs feature. The main improvements are achieved in the airport, shopping mall, metro station, park, pedestrian, street traffic and tram scenes. Special hidden events often occur in these scenes, and these acoustic events are highly related to the background. Therefore, the proposed HACFs can effectively represent the hidden information to improve recognition rate.

We use the basic classifier and complex classifier in each classifier, according to HM-EMD basic characteristics, HMEMD basic features + HACFs features and classic MFCC features are used to classify and recognize the environmental audio stream. The basic classifier is the simple three-layer perceptron shown in Figure 6, while the complex classifier adopts the optimal classifier used in the DCASE competition [24], the classification results are shown in Tables 1, 2. From these two tables, it can be seen that the HM-EMD feature is superior to the MFCC feature with different classifiers: Given the basic classifier, $f_{I F}+A_{I F}$ is 6.7 percentage points higher than that in the MFCC series; after the addition of HACFs, the recognition rate increases by 17.4 percentage points. This result is close to the classification accuracy of the RESNET network with a $32 \mathrm{~m}$ model size in the DCASE competition, while the simple model we used is only $225 \mathrm{~K}$. In a complex classification model, the improvement of model classification can make up for the lack of features to some extent. However, in this case, $f_{I F}+A_{I F}+A H C F s$ still improves the accuracy by $1.3 \%$, and the recognition result reaches $75.7 \%$. This result indicates that the HM-EMD feature provides complete and pure time-frequency domain information, which helps improve the accuracy of
TABLE 1 | Comparison of environmental audio stream classification results based on DCASE dataset.

\begin{tabular}{lccc}
\hline Scene label & MFCC (\%) & $\mathbf{f}_{\mathbf{I F}}+\mathbf{A}_{\mathbf{I F}}$ & $\mathbf{f}_{\mathbf{I F}}+\mathbf{A}_{\mathbf{I F}}+\mathbf{H A C F s}$ \\
\hline Airport & 45.0 & $41.5 \%$ & $51.1 \%$ \\
Bus & 62.9 & $91.4 \%$ & $92.6 \%$ \\
Metro & 53.5 & $93.8 \%$ & $94.1 \%$ \\
Metro Station & 53.5 & $77.2 \%$ & $83.6 \%$ \\
Park & 71.3 & $48.9 \%$ & $72.4 \%$ \\
Public Square & 44.9 & $70.9 \%$ & $72.2 \%$ \\
Shopping Mall & 48.3 & $39.6 \%$ & $55.8 \%$ \\
Street Pedestrian & 29.8 & $44.1 \%$ & $51.1 \%$ \\
Street Traffic & 79.9 & $60.3 \%$ & $76.0 \%$ \\
Tram & 52.2 & $62.4 \%$ & $66.5 \%$ \\
Average & 54.1 & $60.8 \%$ & $71.5 \%$ \\
\hline
\end{tabular}

TABLE 2 | Comparison of classification results of DCASE dataset based on complex classifiers.

\begin{tabular}{lccc}
\hline Classifier & $\begin{array}{c}\text { MFCC } \\
\text { (\%) }\end{array}$ & $\boldsymbol{f}_{\boldsymbol{I F}}+\boldsymbol{A}_{\boldsymbol{I F}}$ & $\boldsymbol{f}_{\boldsymbol{I F}}+\boldsymbol{A}_{\boldsymbol{I F}}+$ HACFs \\
\hline TridentResNet_DevSet & 73.7 & $74.5 \%$ & $75.0 \%$ \\
TridentResNet_EvalSet & 73.7 & $74.5 \%$ & $75.0 \%$ \\
TridentResNet_Ensemble & 74.2 & $75.0 \%$ & $75.5 \%$ \\
TridentResNet_Weighted_Ensemble & 74.4 & $75.2 \%$ & $75.7 \%$
\end{tabular}

the classification of environmental audio streams and proves the effectiveness of the proposed features.

\section{CONCLUSION}

The processing requirements of hidden acoustic signals in ambient audio stream classification are analyzed in this work. Specifically, an ambient audio stream feature extraction method based on HM-EMD is proposed. With the construction of an adaptive mask signal, the frequency domain distribution and IMF dimension are stabilised, and the instability of the time-frequency domain feature system in the acoustic signal processing of the classical EMD algorithm is solved. Then, the time-frequency analysis characteristics of EMD in nonlinear and nonstationary signal processing are fully exploited. Through the Hilbert transform spectrum of each IMF, the hidden components in ambient audio stream signals are analyzed and located to construct the related HACFs. The experimental results show that HM-EMD-based features exhibit greater capability in hidden acoustic event representation than MFCC. Therefore, in our future work, we will study the methods to improve the representation ability of ambient audio streams by exploring the relationship between HM-EMD feature systems and different hidden acoustic events. Attempts will also be made to achieve the accurate labelling of hidden acoustic events in multilevel and multitime scale ambient audio streams with HACFs, such as hidden acoustic event location and hidden acoustic event recognition. In general, the ambient audio stream feature extraction based on HM-EMD represents an effective effort toward ambient audio stream classification. By improving the time-frequency resolution for the analysis of nonstationary environmental acoustic signals, capturing the hidden features of the environment and enhancing the local feature representation, the proposed method can effectively improve the 
efficiency and performance of the classification modelling of the hidden information of ambient audio streams, which provides technical support for camouflaged audio information detection. Hence, it helps to reduce the risk of audio camouflage attacks in social networks.

\section{DATA AVAILABILITY STATEMENT}

The datasets presented in this study can be found in online repositories. The names of the repository/repositories and accession number(s) can be found below: http://dcase. community/challenge2020/task-acoustic-scene-classification.

\section{REFERENCES}

1. Li S, Zhao D, Wu X, Tian Z, Li A, and Wang Z. Functional Immunization of Networks Based on Message Passing. Appl Mathematics Comput (2020). 366: 124728. doi:10.1016/j.amc.2019.124728

2. Han W, Tian Z, and Huang Z, Topic Representation Model Based on Microblogging Behaviour Analysis. World Wide Web. (2020). 23. p. 11-2. doi:10.1007/s11280-020-00822-x

3. Chen T, Kumar A, Nagarsheth P, Sivaraman G, and Khoury E. "Generalization of Audio Deepfake Detection," Odyssey 2020 the Speaker and Language Recognition Workshop. Tokyo, Japan (2020). p. 132-7. doi:10.21437/ Odyssey.2020-19

4. Jati A, Hsu C-C, Pal M, Peri R, AbdAlmageed W, Narayanan S, et al.Adversarial Attack and Defense Strategies for Deep Speaker Recognition Systems. Computer Speech Lang (2021). 68:101199. doi:10.1016/j.csl.2021.101199

5. Al-Turjman F, and Salama R. "Cyber Security in Mobile Social Networks," Security IoT Soc Networks. (2021). p. 55-81. doi:10.1016/b978-0-12-8215999.00003-0

6. Lin ZD, Di CG, and Chen X. Bionic Optimization of MFCC Features Based on Speaker Fast Recognition. Appl Acoust (2020). 173:107682. doi:10.1016/ j.apacoust.2020.107682

7. Sudo Y, Itoyama K, Nishida K, and Nakadai K. Sound Event Aware Environmental Sound Segmentation with Mask U-Net. Adv Robotics (2020). 34(20):1280-90. doi:10.1080/01691864.2020.1829040

8. Waldekar S, and Saha G. Two-level Fusion-Based Acoustic Scene Classification. Appl Acoust (2020). 170:107502. doi:10.1016/j.apacoust.2020.107502

9. Baltrusaitis T, Ahuja C, and Morency LP. Multimodal Machine Learning: A Survey and Taxonomy. IEEE Trans Pattern Anal Mach Intell (2018). 41(2): 423-43. doi:10.1109/TPAMI.2018.2798607

10. Zhang T, and Wu J. Constrained Learned Feature Extraction for Acoustic Scene Classification. Ieee/acm Trans Audio Speech Lang Process (2019). 27(8): 1216-28. doi:10.1109/taslp.2019.2913091

11. Chandrakala S, and Jayalakshmi SL. Generative Model Driven Representation Learning in a Hybrid Framework for Environmental Audio Scene and Sound Event Recognition. IEEE Trans Multimedia (2019). 22(1):3-14. doi:10.1109/ TMM.2019.2925956

12. Xie J, and Zhu M. Investigation of Acoustic and Visual Features for Acoustic Scene Classification. Expert Syst Appl (2019). 126:20-9. doi:10.1016/ j.eswa.2019.01.085

13. Lostanlen V, Lafay G, and Anden J. Relevance-based Quantization of Scattering Features for Unsupervised Mining of Environmental Audio. EURASIP J Audio Speech Music Process (2018). 15. doi:10.1186/s13636-0180138-4

14. Li SD, Jiang LY, and Wu XB. A Weighted Network Community Detection Algorithm Based on Deep Learning. Appl Mathematics Comput (2021). 401: 126012. doi:10.1016/j.amc.2021.126012

15. Suh S, Park S, and Jeong Y. (2020).Designing Acoustic Scene Classification Models with CNN Variants. Dcase2020 Challenge, Tech Rep. Available at: http://dcase.community/documents/challenge2020/technical_reports/ DCASE2020_Suh_101.pdf.

\section{AUTHOR CONTRIBUTIONS}

JL and DZ conceived and designed the study. ZX performed the simulations. ZZ and LY reviewed and edited the manuscript. All authors read and approved the final manuscript.

\section{FUNDING}

This work was supported in part by the N National Key Research and Development Program (2018YFC0830602), and the Sichuan Science and Technology Program (No. 2019YFSY0049).

16. Garcia-Romero D, and McCree A. Stacked Long-Term TDNN for Spoken Language Recognition. In 17th Annual Conference of the InternationalSpeech-Communication-Association (INTERSPEECH 2016). San Francisco, CA, USA (2016). p. 3226-30.

17. Cramer J, Wu HH, and Salamon J. Look, Listen, and Learn More: Design Choices for Deep Audio Embeddings. In ICASSP 2019-2019 IEEE International Conference on Acoustics, Speech and Signal Processing (ICASSP). United Kingdom: Brighton (2019). p. 3852-6.

18. Khan MA, Javed K, Khan SA, Saba T, Habib U, Khan JA, et al. Human Action Recognition Using Fusion of Multiview and Deep Features: an Application to Video Surveillance. Multimed Tools Appl (2020). doi:10.1007/s11042-02008806-9

19. Heittola T, Mesaros A, and Virtanen T. TAU Urban Acoustic Scenes 2020 Mobile, Development Dataset. Dcase2020 Challenge, Tech Rep (2020). doi:10.5281/zenodo.3819968

20. Barbosh M, Singh P, and Sadhu A. Empirical Mode Decomposition and its Variants: A Review With Applications in Structural Health Monitoring. Smart Mater Struct (2020). 29(9):093001. doi:10.1088/ 1361-665X/aba539

21. Kim D, Kim KO, and Oh H-S. Extending the Scope of Empirical Mode Decomposition by Smoothing. EURASIP J Adv Signal Process (2012). 2012: 168. doi:10.1186/1687-6180-2012-168

22. Deering R, and Kaiser JE. The Use of a Masking Signal to Improve Empirical Mode Decomposition. In ICASSP 2005-2005 IEEE International Conference on Acoustics, Speech and Signal Processing (ICASSP). Philadelphia, PA, USA (2005). p. $485-8$.

23. Li H, Li Z, and Mo W. A Time Varying Filter Approach for Empirical Mode Decomposition. Signal Process. (2017). 138:146-58. doi:10.1016/ j.sigpro.2017.03.019

24. Shim HJ, Kim JH, and Jung JW. Audio Tagging and Deep Architectures for Acoustic Scene Classification: Uos Submission for the DCASE 2020 Challenge Dcase2020 Challenge, Tech Rep (2020). Available at: http://dcase.community/ documents/challenge2020/technical_reports/DCASE2020_Shim_120.pdf.

Conflict of Interest: The authors declare that the research was conducted in the absence of any commercial or financial relationships that could be construed as a potential conflict of interest.

Publisher's Note: All claims expressed in this article are solely those of the authors and do not necessarily represent those of their affiliated organizations, or those of the publisher, the editors and the reviewers. Any product that may be evaluated in this article, or claim that may be made by its manufacturer, is not guaranteed or endorsed by the publisher.

Copyright (c) $2021 \mathrm{Lou}, \mathrm{Xu}, \mathrm{Zuo}, \mathrm{Zhang}$ and Ye. This is an open-access article distributed under the terms of the Creative Commons Attribution License (CC BY). The use, distribution or reproduction in other forums is permitted, provided the original author(s) and the copyright owner(s) are credited and that the original publication in this journal is cited, in accordance with accepted academic practice. No use, distribution or reproduction is permitted which does not comply with these terms. 